04

\title{
Влияние вида функции распределения электронов в плазме и дисперсии по энергии электронного пучка на устойчивость низковольтного пучкового разряда
}

\author{
(C) В.С. Сухомлинов, ${ }^{1}$ Р.М. Матвеев, ${ }^{1}$ А.С. Мустафраев, ${ }^{2}$ В.А. Павлов, ${ }^{1}$ С.В. Гордеев ${ }^{1}$
}

${ }^{1}$ Санкт-Петербургский государственный университет, 198504 Санкт-Петербург, Россия

${ }^{2}$ Санкт-Петербургский горный университет, 199106 Санкт-Петербург, Россия

e-mail: v_sukhomlinov@mail.ru

Поступило в Редакцию 9 октября 2020 г.

В окончательной редакции 9 ноября 2020 г.

Принято к публикации 10 ноября 2020 г.

В рамках кинетического подхода исследованы условия потери устойчивости низковольтного пучкового разряда в инертных газах в зависимости от температуры электронного пучка, дисперсии скорости электронов пучка в направлении оси разряда и вида функции распределения электронов по энергиям (ФРЭ). Рассмотрены режимы, когда межэлектродное расстояние порядка длины пробега электронов относительно упругих столкновений с атомами инертного газа. Показано, что температура пучка $T_{b}$, определяемая в низковольтном пучковом разряде температурой катода, не превосходящей $1500 \mathrm{~K}$, и дисперсия энергии электронов пучка, которая в разряде этого типа может быть существенно больше величины $k T_{b}$, ) и достигает $1-2 \mathrm{eV}$, слабо влияют на условия потери устойчивости и величину инкремента усиления возмущений на частотах вплоть до плазменной. Обнаружено, что вид ФРЭ, монотонно падающей с увеличением энергии электронов, также не влияет на параметры распространяющихся в низковольтном пучковом разряде возмущений при энергии пучка много больше средней энергии электронов в плазме. Полученные результаты применимы не только к разряду этого типа, но и другим видам самостоятельных пучковых разрядов.

Ключевые слова: низковольтный пучковый разряд, плазменные неустойчивости, системы электронный пучок-плазма, функция распределения электронов.

DOI: $10.21883 /$ JTF.2021.04.50620.286-20

\section{Введение}

Новые методы и результаты исследований закономерностей динамики релаксации пучков заряженных частиц в плазме [1-4] важны для разработки приборов плазменной электроники нового поколения, в которых применяется пучковая плазма: управляемых стабилизаторов широкого назначения; источников интенсивного электромагнитного излучения; управляемых ключевых элементов электронных цепей; плазмохимических реакторов и т.п. Однако при практическом использовании пучковых разрядов зачастую возникают неустойчивости различного типа, препятствующие их применению в прикладных целях.

Из наиболее распространенных можно выделить плазменно-пучковую неустойчивость, обусловленную резонансным взаимодействием пучка заряженных частиц, движущегося в плазме, с возбуждаемыми им волнами. Теоретически она была предсказана А.И. Ахиезером, Я.Б. Файнбергом [5], а также независимо Д. Бомом, Е. Гроссом [6].

Один из типов разрядов, в котором проявляются эти явления, - низковольтный пучковый разряд в инертных газах (НПР) [7-13]. В работах [7-9] был теоретически и экспериментально исследован НПР в Не для столк- новительного случая, когда длина пробега электрона $\lambda_{\text {ea }}$ относительно упругих столкновений много меньше межэлектродного расстояния $d$.

Для условий, когда $d \sim \lambda_{e a}$, в работах $[12,13]$ в рамках кинетического подхода была рассмотрена задача об устойчивости системы пучок электронов-плазма НПР в предположении моноэнергетического, мононаправленного пучка. В разряде этого типа пучок создается за счет ускорения в прикатодном падении потенциала электронов, испускаемых подогреваемым катодом. В этой ситуации вполне очевидно, что сразу после вылета из катода электроны имеют распределения: изотропное по полусфере „вперед“ и максвелловское по энергиям с температурой катода. Таким образом, функция распределения электронов по энергиям электронов (ФРЭ), испускаемых катодом, после ускорения в прикатодном слое характеризуется тремя параметрами: концентрацией $n_{0 b}$, средней энергией $E_{0 \beta}$ и температурой изотропного распределения пучка по передней полусфере (равной температуре катода) $T_{b}$.

Следует отметить, что ранее влияние ненулевой температуры ФРЭ пучковых электронов на устойчивость системы пучок электронов-плазма в рамках гидродинамического подхода рассматривалась рядом авторов $[14,15]$. При этом считалось, что температура является характе- 
ристикой изотропности ФРЭ пучка по полной сфере. То есть предполагалось, что в пучке есть (в соответствии с максвелловским распределением по скоростям) электроны, движущиеся в том числе и в обратном направлении. Очевидно, что наличие в пучке электронов, которые двигаются против пучка, приводит к росту устойчивости системы пучок-плазма, поскольку эти электроны затрудняют перенос энергии колебаний параметров пучка в направлении его распространения. Отсюда следует, что хотя качественно оба подхода к оценке влияния температуры пучка (рассмотренный другими авторами ранее и применяемый нами в настоящей работе) на устойчивость системы пучок-плазма приводят к ее росту количественное сравнение результатов мало информативно.

Кроме того, во всех работах, посвященных вопросам устойчивости системы высокоэнергетичный слабый пучок-плазма (включая и цитированные выше работы по НПР), ФРЭ плазмы считалась максвеловской. Вполне очевидно, что в зависимости от условий в плазме, особенно в плазме самостоятельного разряда, ФРЭ может существенно отклоняться от равновесной [16]. Данный вопрос в литературе практически не изучен.

Таким образом, актуальным является исследование условий потери устойчивости в НПР при числах Кнудсена разряда порядка единицы в зависимости от вида ФРЭ, наличия в ФРЭ пучка изотропной составляющей, вызванной подогреваемым катодом и дисперсии составляющей скорости в направлении распространения пучка. Этому и посвящена настоящая работа.

\section{1. Получение основных соотношений}

Предположим, что у нас есть НПР с плоскими электродами. При его рассмотрении будем пользоваться физической моделью, развитой в работах $[12,13]$. Согласно этой модели, разряд адекватно описывается бесконечным слоем между плоскостями - катодом и анодом. Введем декартовую систему координат $X Y Z$, плоскость $X Y$ которой совпадает с катодом, а направление оси $Z-$ с внешней нормалью к катоду. В соответствии с развитой моделью можно пренебречь изменением потенциала между границами катодного и анодного слоя, поскольку оно существенно меньше, чем катодное падение $[8,10]$. В условиях, когда число Кнудсена разряда порядка единицы, в НПР можно также не учитывать толщину прикатодного слоя $[11,12]$, которая существенно меньше межэлектродного расстояния, и считать, что пучок с энергией порядка потенциала ионизации атома рабочего газа вылетает из катода (плоскость $z=0$ ).

Для решения поставленной задачи необходимо получить дисперсионное уравнение для малых возмущений в НПР с учетом того факта, что $T_{b} \neq 0$. В соответствии с вышесказанным невозмущенная ФРЭ пучка (т.е. в отсутствие колебаний), нормированная на концентрацию $n_{0 b}$, в условиях, когда $\lambda_{e a} \sim d$, имеет вид

$$
\begin{aligned}
& F_{0 b}(\mathbf{v}, z)=2 \frac{n_{0 b}}{v_{T_{b}}^{3}}\left(\frac{1}{\pi}\right)^{3 / 2} \frac{\exp \left(\frac{-z}{\lambda_{e a}}\right)}{\operatorname{erfc}(1 / \sqrt{\beta})} \\
& \times \exp \left(-\frac{v_{x}^{2}}{v_{T_{b}}^{2}}\right) \exp \left(-\frac{v_{y}^{2}}{v_{T_{b}}^{2}}\right) \exp \left[-\frac{v_{z}^{2}}{v_{T_{b}}^{2}}\right] \theta\left(v_{z}-v_{0}\right),
\end{aligned}
$$

где $\mathbf{v}-$ скорость электрона; $v_{T_{b}}=\sqrt{\frac{2 k T_{b}}{m}} ; k, m, n_{0 b}$ постоянная Больцмана, масса электрона и концентрация электронов в пучке соответственно; $\beta=\left(v_{T_{b}} / v_{0}\right)^{2}$, $v_{0}=\sqrt{\frac{2 E_{0}}{m}}-$ скорость, которую приобретает электрон в катодном слое НПР.

Нетрудно видеть, что при $v_{0} / v_{T_{b}} \rightarrow \infty$ выполняется:

$$
F_{0 b}(\mathbf{v}, z) \rightarrow \frac{1}{2 \pi} \delta\left(v-v_{0}\right) \delta(\mu-1) \exp \left(\frac{-z}{\lambda_{e a}}\right),
$$

где $\mu$ - угол между осью $z$ и скоростью электрона.

Как известно, задачу об устойчивости системы, в которой возможна генерация бегущих монохроматических волн вида $A \cdot \exp [i(\omega t \pm \gamma z)]$ (где $A, \omega, \gamma, t-$ амплитуда, круговая частота, волновое число и время), можно решать в двух постановках: с начальными условиями, когда комплексной полагается частота $\omega$ и ставятся начальные условия для распределения возмущения по объему, где существует рассматриваемая система $[5,9,15,17]$, и с граничными условиями, когда комплексным полагается волновое число и на некоторой поверхности или на ее части (например, на границе системы) ставятся граничные условия, определяющие временное поведение источника колебаний $[12,18,19]$. В первой задаче исследуется временная динамика первоначального возмущения, во втором - пространственная динамика возмущения, распространяющегося от источника.

Дисперсионное уравнение получается из уравнения Пуассона при подставлении в него возмущения плотности заряда, вычисленного на основании кинетических уравнений для возмущений ФРЭ различных групп электронов. Для НПР при числах Кнудсена порядка единицы это три группы: пучковые электроны, электроны, испытавшие упругие столкновения с атомами, имеющие распределение по направлениям движения, близкое к изотропному, и энергию пучковых электронов, а также электроны плазмы $[12,13]$.

В соответствии с результатами этих работ для получения дисперсионного уравнения НПР в приближении пучка постоянной интенсивности (но с учетом упругих электрон - атомных столкновений в кинетических уравнениях для возмущений ФРЭ плазмы, пучка и группы электронов с изотропным распределением по скоростям, имеющих энергию первоначального пучка $\left.E_{0}\right)$ для обеих задач необходимо вычислить выражение 
(см. Приложение):

$$
I_{b}=8 \pi^{2} e \int_{0}^{\infty} \int_{-1}^{1} v^{2} b_{b}^{0}(v, \mu) d \mu d v
$$

где $e-$ заряд электрона; $\bar{v}_{e a}\left(v_{0}\right)=v_{e a}\left(v_{0}\right) \times$ $\times\left[1-\bar{\mu}_{0}\left(v_{0}\right)\right] \quad-$ эффективная частота упругих электрон-атомных столкновений; $v_{e a}\left(v_{0}\right)$ - частота упругих электрон-атомных столкновений; $\bar{\mu}_{0}\left(v_{0}\right)-$ средний косинус угла упругого рассеяния электрона на атоме; функция $b_{b}^{0}(v, \mu)$ определена в Приложении. В [12] для $F_{0 b}$ в виде (2) получено

$$
I_{b}=-i E^{00} \frac{\omega_{b D}^{2}}{\gamma v_{0}^{2} \varepsilon^{2}}
$$

где $E^{00}-$ нулевой член разложения амплитуды возмущения электрического поля по степеням $z$; $\omega_{b D}$ - ленгмюровская частота пучка электронов; $\varepsilon=\frac{\left(\omega-\gamma v_{0}\right)-i \bar{\nu}_{e a}\left(v_{0}\right)}{\gamma v_{0}}$. В случае, когда невозмущенная ФРЭ пучка определяется соотношением (1), после тождественных преобразований получаем

$$
\begin{aligned}
& I_{b}=-i E^{00} \frac{\omega_{b D}^{2}}{\gamma v_{0}^{2}}\left\{\int_{0}^{\infty} \frac{\exp (-x)}{\sqrt{1+\beta x}} d x\right\}^{-1} \\
& \times \int_{0}^{\infty} \frac{\exp (-x)}{\sqrt{1+\beta x}(1+\varepsilon-\sqrt{1+\beta x})^{2}} d x .
\end{aligned}
$$

Необходимо отметить, что, как показано в экспериментах $[10,11]$, в НПР изменение потенциала в области между анодом и внешней границей катодного слоя может быть порядка $1-2 \mathrm{eV}$. Причина этого существование слабого электрического поля, повышающее энергию пучка, и прианодное падение потенциала, которое, напротив, замедляет пучок.

Это означает, что дисперсия распределения по энергиям вдоль оси $z$ пучковых электронов, не испытавших ни одного упругого столкновения с атомами инертного газа, может быть существенно больше, чем дисперсия по энергиям в плоскости $X Y$, составляющая величину порядка температуры катода, т.е. около $0.1 \mathrm{eV}$. Исследуем влияние этого фактора на устойчивость НПР. Если полагать, что распределение по составляющей скорости $v_{z}$ описывается максвелловской функцией с некоторой температурой $T_{z} \neq T_{b}$, то ФРЭ пучка будет по-прежнему описываться соотношением (1) с заменой в его правой части произведения $v_{T_{b}}^{-3} \exp \left(-\frac{v_{z}^{2}}{v_{T_{b}}^{2}}\right)$ на $v_{T_{b}}^{-2} v_{T_{z}}^{-1} \exp \left(-\frac{v_{z}^{2}}{v_{T_{z}}^{2}}\right)$, где $v_{T_{z}}=\sqrt{\frac{2 k T_{z}}{m}}$. Не трудно видеть, что вычисление интеграла $I_{b}$ в этом случае приводит к формуле (5) с заменой $\beta$ на $\beta_{z}=\left(\frac{v_{T z}}{v_{0}}\right)^{2}$. Отметим, что при учете дисперсии ФРЭ пучковых электронов по скоростям увеличивается их средняя энергия, которая в случае ФРЭ пучка, определенной соотношением (2), равна $E_{0}$. Действительно, при ускорении в пристеночном слое вдоль направления $z$ электронов, испускаемых катодом, скорость $v_{0}$ оказывается минимальной составляющей $v_{z}$ пучковых электронов. К этой составляющей добавляются еще и $v_{x},-v_{y}-$ компоненты, которые также распределены с некоторой дисперсией, определяемой параметром $\beta$. Таким образом, в случае, когда ФРЭ пучковых электронов определена формулой (1), $E_{0}$ - минимальная энергия пучковых электронов при $v_{x}=v_{y}=0$.

Рассчитаем среднюю энергию электронов пучка, соответствующую ФРЭ по формуле (1) с учетом сделанного выше замечания о различии дисперсии ФРЭ пучка по скоростям $v_{x}, v_{y}$ и $v_{z}$. При $\sqrt{\beta}, \sqrt{\beta_{z}} \ll 1$ расчеты дают

$$
\begin{aligned}
& E_{0 \beta}=E_{0}\left[1+\beta_{z}+\beta+\beta_{z}^{2}+O\left(\beta_{z}^{3}\right)\right], \\
& \lim _{x \rightarrow 0} \frac{O(x)}{x}=\text { const } \neq 0, \quad \text { const }<\infty .
\end{aligned}
$$

Пользуясь результатами [12], с учетом (5) и (6) из уравнения (П6) Приложения получаем дисперсионное уравнение для задачи с начальными условиями:

$$
\begin{aligned}
& \kappa_{b}\left[\int_{0}^{\infty} \frac{\exp (-x)}{\sqrt{1+\beta_{z} x}} d x\right]_{0}^{-1} \int_{0}^{\infty} \frac{\exp (-x)}{\sqrt{1+\beta_{z} x}\left(1+\varepsilon-\sqrt{1+\beta_{z} x}\right)^{2}} d x \\
& \left.+\left[\frac{1}{(1+\varepsilon)^{2}}+\frac{\kappa_{E} \kappa_{E \beta}}{(1+\varepsilon)^{4}}\right]\left[1+i \frac{v_{e a}}{\gamma v_{0}(1+\varepsilon)}\right)\right]^{-1} \\
& -\frac{\gamma^{2} v_{0}^{2}}{\omega_{D}^{2}}+\frac{\kappa_{i}}{\varepsilon(2+\varepsilon)}\left[1+i \frac{\bar{\nu}_{e a}\left(v_{0}\right)}{2 \gamma v_{0}} \ln \left(\frac{2+\varepsilon}{\varepsilon}\right)\right]^{-1}=0,
\end{aligned}
$$

где $\kappa_{i}=\frac{\omega_{i D}^{2}}{\omega_{D}^{2}} ; \kappa_{b}=\frac{\omega_{b D}^{2}}{\omega_{D}^{2}} ; \kappa_{E}=\frac{E_{t}}{E_{0}} ; \kappa_{E \beta}=\frac{E_{0}}{E_{0 \beta}}$. Для задачи с граничными условиями необходимо выразить неизвестную величину $\gamma$ через известную частоту $\omega$ и переменную $\varepsilon$. Таким образом, получаем дисперсионное уравнение для задачи с граничными условиями в виде:

$$
\begin{aligned}
& \kappa_{b}\left[\int_{0}^{\infty} \frac{\exp (-x)}{\sqrt{1+\beta_{z} x}} d x\right]^{-1} \int_{0}^{\infty} \frac{\exp (-x)}{\left.\sqrt{1+\beta_{z} x}\left(1+\varepsilon-\sqrt{1+\beta_{z} x}\right)\right)^{2}} d x \\
& +\left[\frac{1}{(1+\varepsilon)^{2}}+\frac{\kappa_{E} \kappa_{E \beta}}{(1+\varepsilon)^{4}}\right]\left(1-i \frac{v_{e a}}{\omega_{D} \kappa_{\omega}\left(1-i \frac{\bar{\nu}_{e a}\left(v_{0}\right)}{\kappa_{\omega} \omega_{D}}\right)}\right)^{-1} \\
& -\frac{\left(1-i \frac{1}{\kappa_{\omega}} \frac{v_{e a}\left(v_{0}\right)}{\omega_{D}}\right)^{2}}{(1+\varepsilon)^{2}} \kappa_{\omega}^{2}+\frac{\kappa_{i}}{\varepsilon(2+\varepsilon)} \\
& \times\left[1+i \frac{\nu_{e a}\left(v_{0}\right)(1+\varepsilon)}{2 \omega_{D} \kappa_{\omega}\left(1-i \frac{1}{\kappa_{\omega}} \frac{\bar{\nu}_{e a}\left(v_{0}\right)}{\omega_{D}}\right)} \ln \left(\frac{2+\varepsilon}{\varepsilon}\right)\right]^{-1}=0,
\end{aligned}
$$


где $E_{t}-$ средняя энергия электронов плазмы; $\kappa_{\omega}=\frac{\omega}{\omega_{D}} ; \nu_{e a}-$ частота упругих электрон-атомных столкновений, которая считается не зависящей от относительной скорости сталкивающихся частиц, $v_{\beta e a}\left(v_{0}\right)=v_{e a}\left[1-\bar{\mu}\left(v_{0 \beta}\right)\right] ; \bar{\mu}\left(v_{0 \beta}\right)-$ средний косинус угла упругого рассеяния электрона с энергией $E_{0 \beta}=\frac{m v_{0 \beta}^{2}}{2}$ на атоме; $\omega_{D}, \omega_{i D}, \omega_{b D}-$ плазменные частоты собственно плазмы, группы электронов с изотропным распределением и энергией пучка и самого пучка, соответственно.

Нетрудно показать, что при $\beta \rightarrow 0$ из (7) и (8) мы получим уравнения (18) и (25), соответственно, работы [12].

Таким образом, при оценке влияния дисперсии скорости $v_{z}$ электронов пучка из-за непостоянства потенциала между катодным слоем и анодом в НПР нужно пользоваться дисперсионными уравнениями (7) и (8) для задач с начальными и граничными условиями, соответственно.

Напомним, что для задачи с начальными условиями, (при выбранном нами знаке в показателе экспоненты, описывающей гармоническое возмущение) ищется решение уравнения (7) $\varepsilon_{n}$, удовлетворяющее неравенству:

$$
\operatorname{Im}\left(\varepsilon_{n}\right) \leq 0
$$

При этом относительные инкремент и сдвиг частоты возмущения связаны с $\varepsilon_{n}$ соотношениями соответственно:

$$
\frac{\operatorname{Im}(\omega)}{\gamma v_{0}}=\operatorname{Im}\left(\varepsilon_{n}\right)+\frac{\bar{\nu}_{e a}\left(v_{0}\right)}{\gamma v_{0}} ; \quad \frac{\operatorname{Re}(\omega)}{\gamma v_{0}}=1+\operatorname{Re}\left(\varepsilon_{n}\right) .
$$

Для задачи с граничными условиями аналогично ищется решение $\varepsilon_{n}$ уравнения (8), удовлетворяющее неравенству (9). Условия потери устойчивости для задач с начальными и граничными условиями при выбранном нами знаке в показателе экспоненты для бегущей волны возмущения имеют вид, соответственно:

$$
\operatorname{Im}(\omega)<0 ; \quad \operatorname{Im}\left(\gamma v_{0}\right)>0
$$

Рассмотрим теперь влияние вида ФРЭ плазмы на устойчивость системы пучок-плазма на примере НПР. Как правило, при рассмотрении системы пучок-плазма (в том числе и в НПР) полагается, что электроны плазмы распределены в соответствие с максвелловской ФРЭ. Однако экспериментальные исследования НПР показывают, что реально ФРЭ может существенно от нее отклоняться [9-11]. Возникает вопрос насколько при этом изменяются условия потери устойчивости системы электронный пучок-плазма, каковы инкремент, сдвиг частоты, фазовая и групповая скорости распространения малых возмущений.

Необходимо отметить, что ФРЭ в плазме НПР при рассматриваемых условиях может не являться монотонной. Например, для плазмы Не при энергиях 20-21 и $24.4 \mathrm{eV}$ наблюдаются пики ФРЭ, вызванные девозбуждением атомов метастабильных атомов $\operatorname{He}\left(2^{3} S_{1}\right)$ и их ионизацией электронами пучка соответственно [11]. Однако их концентрация не превосходит одного процента от концентрации электронов в пучке и не влияет на условия потери устойчивости НПР. Поэтому далее мы будем считать ФРЭ в плазме НПР монотонно падающей.

Для исследования сформулированной выше проблемы рассмотрим некоторую произвольную изотропную по скоростям и не зависящую от координаты $z$ ФРЭ в плазме $F_{0 t}(v)$, которая удовлетворяет условию нормировки:

$$
4 \pi \int_{0}^{\infty} v^{2} F_{0 t}(v) d v=n_{0 t},
$$

где $n_{0 t}-$ концентрация электронов в плазме НПР. При этом пусть средняя энергия электронов в плазме равна $E_{t}$.

Соответствующий плазме член в дисперсионном уравнении, согласно [12], пропорционален величине $I_{t}$ (см. Приложение):

$$
I_{t}=8 \pi^{2} e \int_{0}^{\infty} \int_{-1}^{1} v^{2} b_{t}(v, \mu) d \mu d v
$$

где величина $b_{t}(v, \mu)$ определена в Приложении. Если $F_{0 t}$ изотропна, то это соотношение можно переписать в виде

$$
I_{t}=i \frac{8 \pi^{2} e}{m \gamma v_{0}(1+\varepsilon)} \int_{-1}^{1} \mu d \mu \int_{0}^{\infty} \frac{v^{2}}{\left[1-\frac{\mu v}{v_{0}(1+\varepsilon)}\right]} \frac{d F_{0 t}}{d v} d v
$$

Для ограниченности интеграла (14) необходимо и достаточно, чтобы выполнялось соотношение

$$
\lim _{v \rightarrow \infty} \frac{d F_{0 t}}{d v}=o\left(v^{-1}\right)
$$

где $\lim _{x \rightarrow 0} \frac{o(x)}{x}=0$, а знаменатель подынтегрального выражения не имел корней на действительной оси.

При вычислении интеграла (14) необходимо учитывать, что знаменатель подынтегрального выражения имеет комплексный корень. В плазме при отсутствии пучка быстрых электронов это является следствием бесстолкновительного затухания Ландау [20], которое необходимо учитывать, когда фазовая скорость распространения возмущений становится порядка средней тепловой скорости электронов. Можно показать, что при наличии пучка быстрых электронов, скорость которых существенно выше тепловой скорости электронов плазмы, достаточно быстром спаде $F_{0 t}(v)$ при $v \rightarrow \infty$ (см. соотношение (15)), а также учете упругих электронатомных столкновений, этим затуханием можно пренебречь. Таким образом, вычисление интеграла (14) сводится к расчету его главного значения по Коши.

Предположим, что при любом целом $j>0$ выполняется равенство

$$
\lim _{v \rightarrow \infty} v^{j} \frac{d F_{0 t}}{d v}=0
$$


Это соотношение верно для большинства практически реализуемых ФРЭ в изотропной плазме (максвелловской, дрювистейновской и т.д.).

Тогда для вычисления главного значения по Коши интеграла в (14) можно разложить функцию $\frac{1}{1-\frac{\mu v}{v_{0}(1+\varepsilon)}}$ в ряд Тейлора. При этом после интегрирования по переменной $\mu$ четные члены разложения зануляются. Таким образом, получаем

$$
\begin{aligned}
I_{t}=-i \frac{\omega_{D}^{2}}{\gamma v_{0}^{2}}\left[\frac{1}{(1+\varepsilon)^{2}}+\frac{1}{(1+\varepsilon)^{4}} \frac{E_{t}}{E_{0}}+O\left(\frac{\overline{E_{t}^{2}}}{E_{0}^{2}}\right)\right], \\
\frac{E_{t}}{E_{0}} \ll 1,
\end{aligned}
$$

где $\omega_{D}^{2}=\frac{4 \pi^{2} e n_{0 t}}{m}, \bar{E}_{t}^{2}-$ среднее значение величины $E_{t}^{2}$ в плазме. Тогда второй член в дисперсионных уравнениях (7), (8) для величины $I_{t}$, определенной формулой (14), приобретает вид

$$
\begin{aligned}
& {\left[\frac{1}{(1+\varepsilon)^{2}}+\frac{1}{(1+\varepsilon)^{4}} \frac{E_{t}}{E_{0}}+O\left(\overline{\frac{E_{t}^{2}}{E_{0}^{2}}}\right)\right]} \\
& \times\left[1+i \frac{v_{e a}}{\gamma v_{0}(1+\varepsilon)}\right]^{-1}, \\
& {\left[\frac{1}{(1+\varepsilon)^{2}}+\frac{1}{(1+\varepsilon)^{4}} \frac{E_{t}}{E_{0}}+O\left(\frac{\overline{E_{t}^{2}}}{E_{0}^{2}}\right)\right]} \\
& \times\left(1-i \frac{v_{e a}}{\omega_{D} \kappa_{\omega}\left(1-i \frac{\bar{v}_{e a}\left(v_{0}\right)}{\kappa_{\omega} \omega_{D}}\right)}\right)^{-1},
\end{aligned}
$$

соответственно. Эти соотношения тождественно совпадают с соответствующими выражениями, полученными для максвелловской ФРЭ в работе [12].

Отсюда следует, что вид дисперсионного уравнения при $\frac{E_{t}}{E_{0}} \ll 1$ зависит в основном от средней энергии электронов плазмы, но не от вида ФРЭ.

\section{2. Обсуждение полученных результатов}

Рассмотрим зависимость условий потери устойчивости системы плазмы НПР от температуры катода, влияния прианодного падения потенциала в анодном слое и слабого электрического поля между катодным и анодными слоями в НПР. Типичные значения температуры катода при экспериментах в инертных газах $\mathrm{He}, \mathrm{Ne}, \mathrm{Ar}$ обычно не превышают $1500 \mathrm{~K}$ [9-11]. Величина прианодного падения в рассматриваемых условиях в НПР имеет величину порядка $1-2 \mathrm{eV}[11]$. Наконец, изменение потенциала между катодным и анодным слоями также имеет порядок $1-2 \mathrm{eV}$ [11]. Таким образом, можно считать, что, например, для НПР в Не параметр $\beta_{z} \approx \frac{\Delta E}{E_{0}}$, где $\Delta E<2 \mathrm{eV}, E_{0} \simeq 30 \mathrm{eV}$, при рассматриваемых условиях не превосходит величины 0.03-0.07. При этом такие сравнительно большие значения обусловлены в основном прианодным падением потенциала и наличием слабого электрического поля между катодным слоем и анодом, а вклад температуры катода пренебрежимо мал.

Для примера мы рассмотрим задачу с граничными условиями, поскольку, как показано в работах [12,13], именно такая постановка соответствует реальной ситуации в НПР.

Прежде всего отметим, что расчеты при параметрах $\beta, \beta_{z}$, соответствующих температуре катода в НПР (см. выше), показывают, что характеристики возмущений (инкремент, скорость распространения и т.п.) на любых частотах вплоть до плазменной от этой температуры не зависят. Это обусловлено сравнительно низкой по сравнению с энергий пучка в НПР величиной тепловой энергии, соответствующей этой температуре. Поэтому далее мы рассмотрим зависимость только от параметра $\beta_{z}$, который в плазме НПР может изменяться в более широком диапазоне (см. выше).

На рис. 1 приведены результаты расчета зависимостей относительного инкремента $\frac{\operatorname{Im}\left(\gamma v_{0}\right.}{\omega_{D}}$ от параметра $\beta_{z}$ в диапазоне $\beta_{z} \leq 1$ (напомним, что реальный диапазон, например, для НПР в Не составляет $\left.\beta_{z} 0.03-0.07\right)$ на основе решения уравнения (8) при различных относительных частотах $\kappa_{\omega}=\frac{\omega}{\omega_{D}}=0.4 ; 0.7 ; 1$ и $\kappa_{i}=\kappa_{b}=0.05$; $\kappa_{E}=\frac{\bar{y}_{e a}}{\omega_{D}}=0.02$, характерных для НПР. Видно, что инкремент сравнительно слабо падает с ростом $\beta_{z}$ при всех рассмотренных относительных частотах $\frac{\omega}{\omega_{D}}$. Это, повидимому, связано с тем, что при увеличении $\beta_{z}$ растет количество электронов пучка с энергией, существенно превосходящей величину $E_{0}$. Поскольку возмущения концентрации электронов вдоль пучка из-за малости его

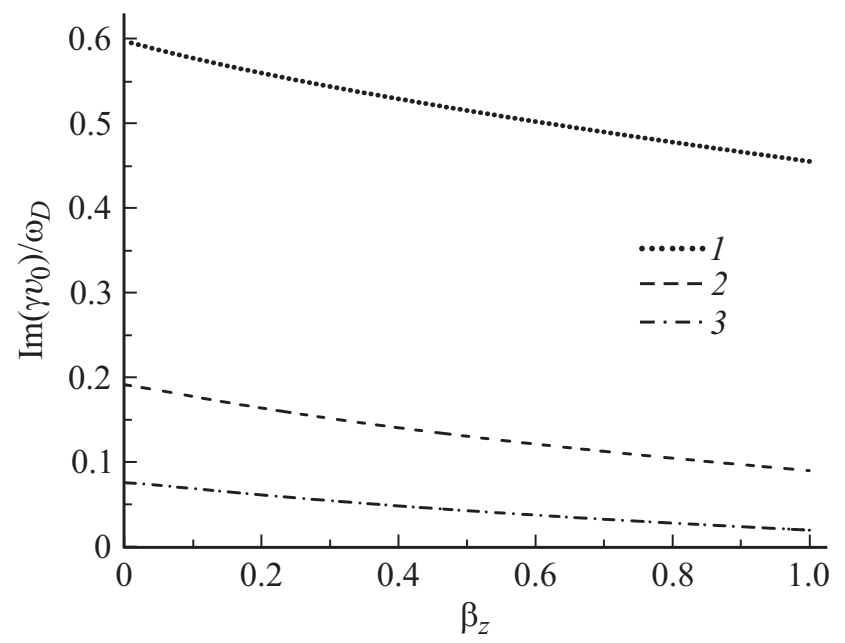

Рис. 1. Зависимость относительного инкремента возмущений в задаче с граничными условиями от параметра $\beta_{z}$ при различных относительных частотах $\kappa_{\omega}: 1-1,2-0.7,3-0.4$; $\kappa_{i}=\kappa_{b}=0.05 ; \kappa_{E}=\frac{\bar{\nu}_{e a}}{\omega_{D}}=0.02$. 


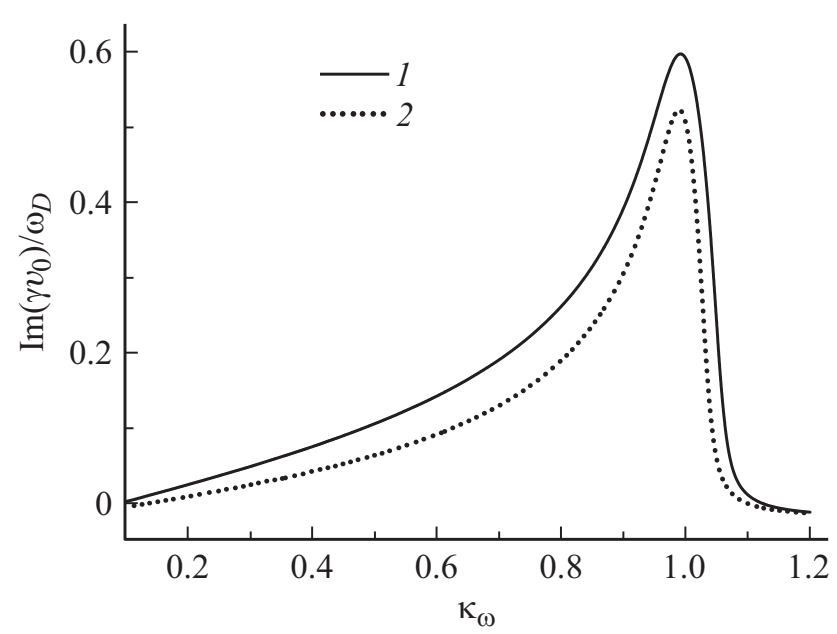

Рис. 2. Зависимость относительного инкремента возмущений в задаче с граничными условиями от относительной частоты при различных параметрах $\beta_{z}: 1-0,2-0.5 ; \kappa_{i}=\kappa_{b}=0.05$; $\kappa_{E}=\frac{\bar{\nu}_{e a}}{\omega_{D}}=0.02$

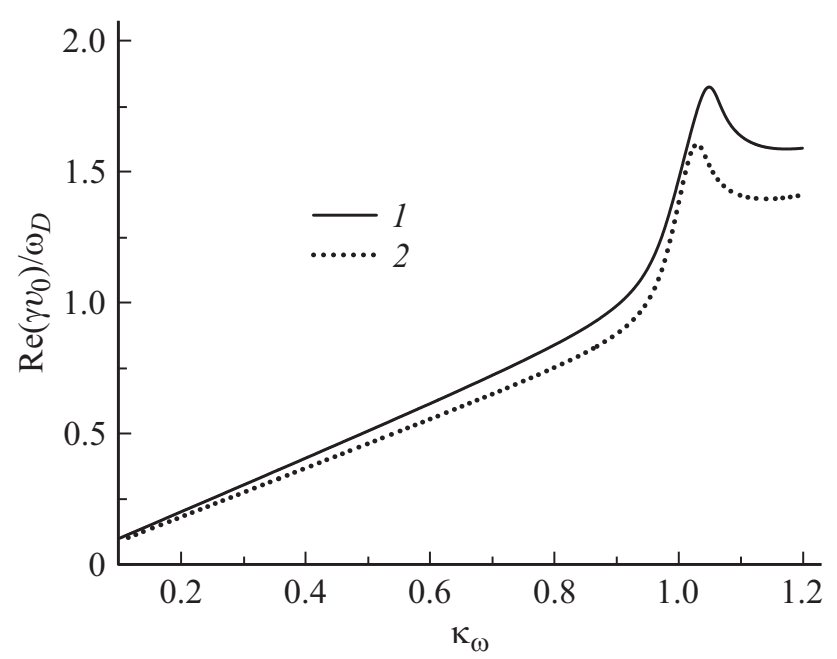

Рис. 3. Зависимость относительного волнового числа возмущений в задаче с граничными условиями от относительной частоты при различных параметрах $\beta_{z}: 1-0,2-0.5$; $\kappa_{i}=\kappa_{b}=0.05 ; \kappa_{E}=\frac{v_{e a}^{-}}{\omega_{D}}=0.02$.

плотности распространяются преимущественно за счет сноса со скоростью самих электронов пучка, то из-за различной скорости электронов растет геометрический размер области существования возмущения, что уменьшает объемную плотность его энергии, а значит, падает скорость его нарастания, т. е. инкремент. При этом в области значений параметра $\beta_{z} \leq 0.03-0.07$, характерного для НПР в Не, изменение инкремента пренебрежимо мало. Забегая вперед, отметим, что это верно и для всех других параметров возмущений (волнового числа, фазовой и групповой скоростей распространения и т.д.).

На рис. 2 показаны рассчитанные зависимости относительного инкремента $\frac{\operatorname{Im}\left(\gamma v_{0}\right)}{\omega_{D}}$ от относительной частоты возмущения $\frac{\omega}{\omega_{D}}$ при различных величинах параметра $\beta_{z}=0 ; 0.5$ и $\kappa_{i}=\kappa_{b}=0.05 ; \kappa_{E}=\frac{\bar{\nu}_{e a}}{\omega_{D}}=0.02$. Видно, что характер обеих зависимостей схожий с четко выраженным максимумом в области плазменной частоты. При частотах $\omega$, больших плазменной, рассчитанные инкременты быстро уменьшаются и стремятся к нулю. Это находится в соответствии с известным фактом быстрого затухания возмущений в плазме при росте волнового числа при частотах выше ленгмюровской [21]. $\mathrm{B}$ соответствие с данными рис. 1 при увеличении $\beta_{z}$ инкремент падает при любых частотах возмущения. Причины этого изложены выше.

Аналогичные зависимости для относительного волнового числа в задаче с граничными условиями при тех же параметрах системы плазмы - электронный пучок представлены на рис. 3. Как видно данных, с ростом частоты наблюдается возрастание волнового числа возмущения. Как мы увидим в дальнейшем, это вызвано соответствующей зависимостью от частоты фазовой скорости распространения возмущения в плазме.

Данные, представленные на рис. 4, 5, свидетельствуют о слабо растущей зависимости фазовой $v_{f}$ и групповой $v_{g}$ скоростей соответственно от параметра $\beta_{z}$. На рис. 5 видна характерная особенность - групповая скорость при $\frac{\omega}{\omega_{D}}=1$ практически не зависит от параметра $\beta_{z}$ и существенно ниже, чем эта величина для частот, меньших плазменной.

Обсудим это подробнее. Как известно, групповая скорость возмущений, распространяющихся на частоте плазмы, определяется только средней тепловой скоростью электронов плазмы [21]. Но в рассматриваемом случае эта величина не зависит от параметра $\beta_{z}$. При частотах $\omega<\omega_{D}$ колебания в плазме не распространяются. Таким образом, перенос возмущений вдоль пучка в этом случае происходит только за счет их сноса в

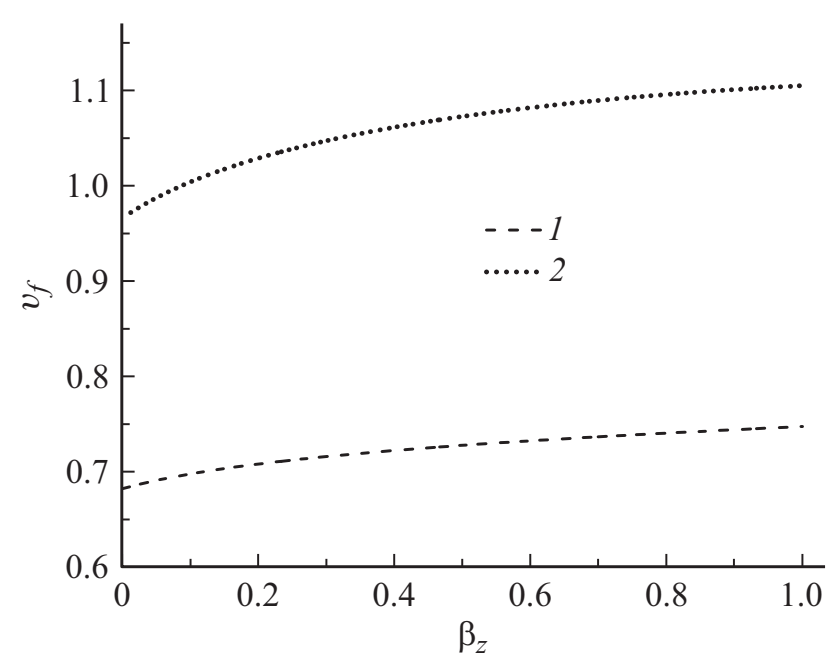

Рис. 4. Зависимость относительной фазовой скорости распространения возмущений $v_{f}$ в задаче с граничными условиями от параметра $\beta_{z}$ при различных относительных частотах $\kappa_{\omega}$ : $1-1,2-0.4 ; \kappa_{i}=\kappa_{b}=0.05 ; \kappa_{E}=\frac{v_{e a}^{-}}{\omega_{D}}=0.02$. 


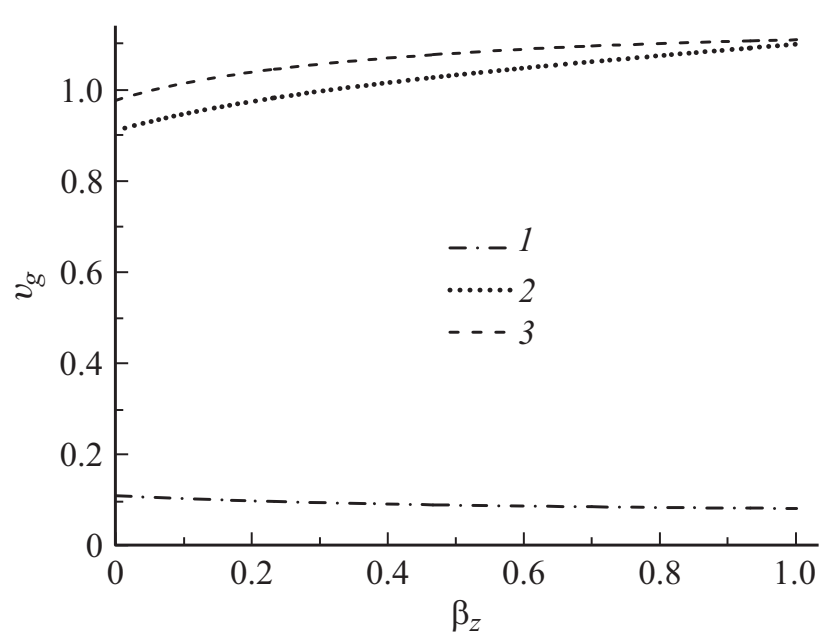

Рис. 5. Зависимость относительной групповой скорости распространения возмущений $v_{f}$ в задаче с граничными условиями от параметра $\beta_{z}$ при различных относительных частотах $\kappa_{\omega}$ : $1-1,2-0.4,3-0.7 ; \kappa_{i}=\kappa_{b}=0.05 ; \kappa_{E}=\frac{v_{e a}^{-}}{\omega_{D}}=0.02$.

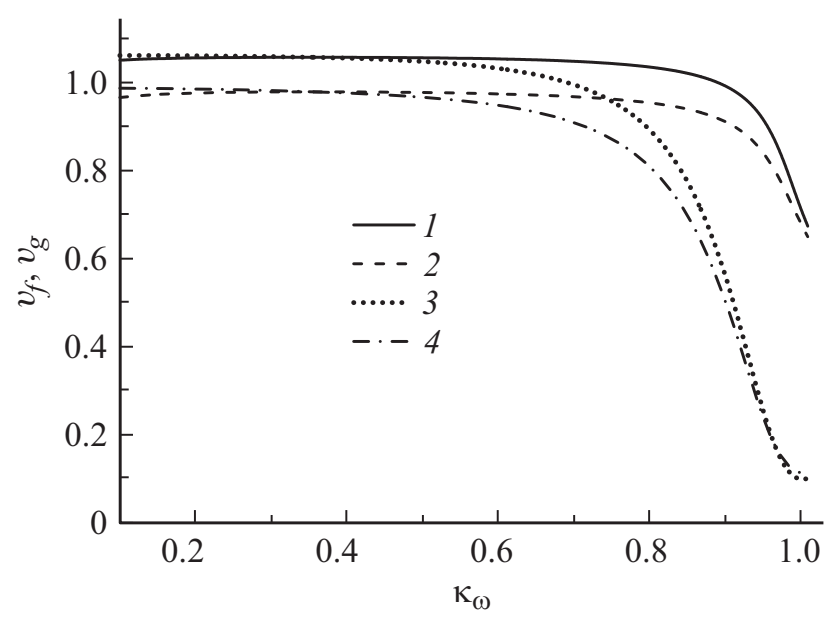

Рис. 6. Зависимость фазовой $v_{f}$ и групповой $v_{g}$ скоростей распространения возмущений в задаче с граничными условиями от относительной частоты при различных параметрах $\beta_{z}$ : $1-v_{f}$ при $\beta_{z}=0.3 ; 2-v_{f}$ при $\beta_{z}=0 ; 3-v_{g}$ при $\beta_{z}=0.3$; 4 -- $v_{g}$ при $\beta_{z}=0 ; \kappa_{i}=\kappa_{b}=0.05 ; \kappa_{E}=\frac{v_{e a}^{-}}{\omega_{D}}=0.02$.

результате движения пучковых электронов. При увеличении параметра $\beta_{z}$ растет число электронов со скоростями, превышающими $v_{0}$. Это и является причиной незначительного роста величин $v_{f}, v_{g}$ с увеличением $\beta_{z}$ при $\frac{\omega}{\omega_{D}}<1$. Данный рост приводит к тому, что при некотором значении $\beta_{z}$ фазовая и/или групповая скорости начинают превышать величину $v_{0}-$ минимальную скорость пучковых электронов.

Зависимости скоростей $v_{f}, v_{g}$ от частоты гармонического возмущения при $\beta_{z}=0 ; 0.3$ представлены на рис. 6. Видно, что в соответствии с ранее сказанным при $\omega \omega_{D}<1$ с ростом $\beta_{z}$ скорости увеличиваются. При $\frac{\omega}{\omega_{D}} \approx 1$ фазовая скорость при $\beta_{z}=0.3$ больше, чем при $\beta_{z}=0$. Однако групповая скорость возмущения на частоте плазмы от параметра $\beta_{z}$ не зависит (причины этого были указаны выше).

\section{Заключение}

При проведении исследований мы использовали результаты, полученные в работах $[12,13]$ для НПР. В частности, расчеты проводились нами для дисперсионных уравнений (7), (8). Они отличаются от обычно используемых при исследовании неустойчивости систем электронный пучок-плазма (см., например, [5,14,15]). Связано это с тем, что в рассматриваемых условиях, когда межэлектродное расстояние (т.е. размеры плазмы) порядка длины пробега электрона относительно упругого столкновения с атомом, в НПР присутствует три группы электронов: пучка, плазмы и электронов с изотропным распределением по скоростям и энергией пучка. Последняя группа при числах Кнудсена разряда порядка единицы образуется в результате нескольких (до трех-четырех) упругих столкновений электронов с атомами инертного газа. Кроме того, необходимо также учитывать упругие электрон-атомные столкновения для всех трех групп электронов. В результате дисперсионное уравнение для НПР, во-первых, содержит член, пропорциональный параметру $\kappa_{i}$, и, во-вторых, учет электрон-атомных столкновений для электронов пучка и изотропной группы высокоскоростных электронов также приводит к существенным отличиям уравнений (7), (8) от обычно используемых для системы пучок электронов-плазма. В этих уравнениях, как правило, авторы либо не учитывали электрон-атомные столкновения совсем [5,6,22-32], либо учитывали их только в плазме $[14,15,33]$.

Необходимо также отметить, что в НПР разность потенциалов анода и катода не является фиксированной, зависит от условий в разряде и не сохраняется при потере устойчивости разряда $[12,13]$. Это характерно для самостоятельных разрядов с отрицательным или малым положительным дифференциальным сопротивлением, для реализации которых используется балластное сопротивление.

Как показывают расчеты, основные выводы о зависимости параметров возмущений в области потери неустойчивости от температуры пучка и дисперсии скорости при $\kappa_{i}=0$ сохраняют свою силу.

Исследование влияния вида ФРЭ на вид дисперсионного уравнения системы электронный пучок-плазма НПР мы проводили в самом общем виде, не используя специфические особенности НПР. При этом быстрые электроны были выделены в отдельные группы (пучковые и изотропные по направлениям движения после упругих электрон-атомных столкновений). Таким образом, неявно предполагалось, что ФРЭ в плазме монотонно спадает при $v \rightarrow \infty$. В этих условиях, как указывалось, вид дисперсионного уравнения при $\frac{E_{t}}{E_{0}} \ll 1$ 
зависит в основном от средней энергии электронов плазмы, но не от вида ФРЭ.

Таким образом, можно утверждать, что с известными оговорками полученные результаты при числах Кнудсена порядка единицы носят общий характер и верны не только для НПР, но и для других типов самостоятельных пучковых разрядов.

\section{Приложение}

Как указывалось, в отсутствие возмущений ФРЭ в НПР при рассматриваемых условиях представляется в виде суммы функций трех групп электронов: первоначального пучка $F_{0 b}(z, \mathbf{v})$, определенной формулой (1) (в работе [12] использовалось выражение (2)); изотропно распределенных электронов с энергией пучка $F_{0 b}^{i}(z, \mathbf{v})$ соответственно, и электронов плазмы $F_{0 t}(z, \mathbf{v})$.

Полные ФРЭ и электрическое поле в НПР представляются в виде суммы невозмущенных (П1) и гармонических возмущений с амплитудами, зависящими от координаты:

$$
\begin{gathered}
F_{b}(z, t, \mathbf{v})=F_{0 b}(z, \mathbf{v})+f_{b}(z, t, \mathbf{v}) \\
F_{b}(z, t, \mathbf{v})=f_{o b}(\mathbf{v}, z) \exp \left[-\sum_{e a}(v) z\right] \cdot \exp [i(\omega t-\gamma z)] \\
F_{b}^{i}(z, t, \mathbf{v})=F_{0 b}^{i}(z, \mathbf{v})+f_{b}^{i}(z, t, \mathbf{v}) \\
f_{b}^{i}(z, t, \mathbf{v})=f_{0 b}^{i}(\mathbf{v}, z) \cdot \exp [i(\omega t-\gamma z)] \\
F_{t}(z, t, \mathbf{v})=F_{0 t}(z, \mathbf{v})+f_{t}(z, t, \mathbf{v}) \\
f_{t}(z, t, \mathbf{v})=f_{o t}(\mathbf{v}, z) \cdot \exp \left[i\left(\omega_{t}-\gamma z\right)\right] \\
\mathbf{E}_{p}=\mathbf{E}_{0 p}(z)+\mathbf{E} ; \quad \mathbf{E}=\mathbf{E}^{0} \cdot \exp [i(\omega t-\gamma z)] \\
\sum_{e a}(v)=\frac{1}{\lambda_{e a}(v)}
\end{gathered}
$$

Из кинетических уравнений для ФРЭ и уравнения Пуассона для амплитуд возмущений имеем систему [12]:

$$
\begin{gathered}
f_{o t}+\frac{v_{z}}{i\left(\omega-\gamma v_{z}\right) v_{e a}^{-}(v)} \frac{\partial f_{0 t}}{\partial z} \\
=\frac{a_{t}(v, \mu)}{2} \int_{-1}^{1} f_{o t}\left(v, \mu^{\prime}, z\right) d \mu^{\prime}+b_{t}(\mu, z), \quad(\Pi 2) \\
f_{o b}^{i}+\frac{v_{z}}{i\left(\omega-\gamma v_{z}\right)+\bar{v}_{e a}(v)} \frac{\partial f_{o b}^{i}}{\partial z} \\
=\frac{a_{b}^{i}(v, \mu)}{2} \int_{-1}^{1} f_{o b}^{i}\left(v, \mu^{\prime}, z\right) d \mu^{\prime}+b_{b}^{i}(\mu, z), \\
\frac{\partial E^{0}}{\partial z}-i \gamma E^{0}=4 \pi e\left\{\int_{o b}=b_{t}(\mu, z),\right. \\
\left.f_{0 t} d^{3} v+\int f_{o b}^{i} d^{3} v+\int f_{0 t} d^{3} v\right\}
\end{gathered}
$$

где

$$
\begin{gathered}
a_{t}=a_{b}^{i}=\frac{\bar{v}_{e a}(v)}{i\left(\omega-\gamma v_{z}\right)+\bar{v}_{e a}(v)} ; \\
b_{t}=-\frac{e E^{0}(z)}{m\left[i\left(\omega-\gamma v_{z}\right)+\bar{v}_{e a}(v)\right]} \frac{\partial F_{0 t}}{\partial v_{z}}, \\
b_{b}^{i}=-\frac{e E^{0}(z)}{m\left[i\left(\omega-\gamma v_{z}\right)+\bar{v}_{e a}(v)\right]} \frac{F_{0 b}^{i}}{\partial v_{z}} ; \\
b_{b}=-\frac{e E^{0}(z)}{m\left[i\left(\omega-\gamma v_{z}\right)+\bar{v}_{e a}(v)\right]} \frac{\partial F_{0 b}}{\partial v_{z}} .
\end{gathered}
$$

Для примера рассмотрим случай НПР в Не. Поскольку частота $v_{e a}$ упругих столкновений электрона с атомом Не слабо зависит от относительной скорости сталкивающихся частиц, то зависимость эффективной частоты $\bar{v}_{e a}(v)$ от скорости $v$ в данном случае обусловлена только зависимостью от этой величины среднего косинуса угла рассеяния. Тогда в уравнениях для $f_{0 b}^{i}, f_{0 b}$ следует, очевидно, положить $\bar{v}_{e a}(v)=\bar{v}_{e a}\left(v_{0}\right)$, а в уравнении для $f_{0 t}-\bar{v}_{e a}(v) \approx \bar{v}_{e a}\left(v_{T}\right)$, где $v_{T}-$ средняя скорость тепловых электронов плазмы. При этом в первом приближении можно считать, что $\bar{\mu}_{0}\left(v_{T}\right)=0$ [34] и, следовательно, $\bar{v}_{e a}\left(v_{T}\right)=v_{e a}$.

Поскольку мы рассматриваем условия, при которых $\lambda_{\text {ea }} \sim d$, то это позволяет искать решение для амплитуд возмущения ФРЭ и поля в виде рядов по степеням $z$, которые будут, в известной степени, быстро сходиться. Разлагая также в аналогичные ряды концентрации $n_{0 b}^{i}(z), n_{0 t}(z)$, получаем

$$
\begin{gathered}
f_{0 t}(z)=\sum_{n=0}^{N} f_{0 t}^{n} z^{n} ; \quad f_{0 b}(z)=\sum_{n=0}^{N} f_{0 b}^{n} z^{n} ; \\
f_{0 b}^{i}(z)=\sum_{n=0}^{N} f_{0 b}^{i n} z^{n}, \\
E^{0}(z)=\sum_{n=0}^{N} E^{0 n} z^{n} ; \quad n_{0 b}^{i}(z)=\sum_{n=0}^{N} n_{0 b}^{i n} z^{n} ; \\
n_{0 t}(z)=\sum_{n=0}^{N} n_{0 t}^{n} z^{n} .
\end{gathered}
$$

Подставляя (П3) и (3) в (П2) и приравнивая коэффициенты при одинаковых степенях $\mathrm{z}$ к нулю, имеем для коэффициентов при $n=j \leq(N-1)$ систему уравнений:

$$
\begin{aligned}
f_{0 t}^{j} & +\frac{v_{z}(j+1) f_{0 t}^{j+1}}{i\left(\omega-\gamma v_{z}\right)+\bar{v}_{e a}\left(v_{T}\right.} \\
& =\frac{a_{t}(v, \mu)}{2} \int_{-1}^{1} f_{0 t}^{j}\left(v, \mu^{\prime}, z\right) d \mu^{\prime}+b_{t}^{j}(\mu), \\
f_{0 b}^{i j}+\frac{v_{z}(j+1) f_{0 t}^{i j+1}}{i\left(\omega-\gamma v_{z}\right)+\bar{v}_{e a}\left(v_{0}\right.} & \\
& =\frac{a_{b}^{i}(v, \mu)}{2} \int_{-1}^{1} f_{0 t}^{i j}\left(v, \mu^{\prime}, z\right) d \mu^{\prime}+b_{b}^{i j}(\mu),
\end{aligned}
$$




$$
\begin{gathered}
f_{0 b}^{j}=b_{b}^{j}(\mu), \\
(j+1) E^{0 j+1}-i \gamma E^{0 j} \\
=4 \pi e\left\{\int f_{0 t}^{j} d^{3} v+\int f_{0 b}^{i j} d^{3} v+\int f_{0 b}^{j} d^{3} v\right\} .
\end{gathered}
$$

Для $j=N$ имеем

$$
\begin{gathered}
f_{0 t}^{N}=\frac{a_{t}(v, \mu)}{2} \int_{-1}^{1} f_{0 t}^{N}\left(v, \mu^{\prime}, z\right) d \mu^{\prime}+b_{t}^{N}(\mu), \\
f_{0 b}^{i N}=\frac{a_{b}^{i}(v, \mu)}{2} \int_{-1}^{1} f_{0 b}^{i N}\left(v, \mu^{\prime}, z\right) d \mu^{\prime}+b_{b}^{i N}(\mu) ; \\
f_{0 b}^{N}=b_{b}^{N}, \\
-i \gamma E^{0 N}=4 \pi e\left\{\int f_{0 t}^{N} d^{3} v+\int f_{0 b}^{i N} d^{3} v+\int f_{0 b}^{N} d^{3} v\right\},
\end{gathered}
$$

при этом, при любом $j \leq N$ выполняется

$$
\begin{aligned}
b_{t}^{j}= & -\frac{e \frac{\partial F_{0,}}{\partial v_{z}}}{m\left[i\left(\omega-\gamma v_{z}\right)+\bar{v}_{e a}\left(v_{T}\right)\right]} \sum_{k=0}^{j} E^{0 k} \frac{n_{0 t}^{j-k}}{n_{0 t}^{0}}, \\
b_{t}^{i j}= & -\frac{e \frac{\partial F_{0 t}}{\partial v_{z}}}{m\left[i\left(\omega-\gamma v_{z}\right)+\bar{v}_{e a}\left(v_{0}\right)\right]} \sum_{k=0}^{j} E^{0 k} \frac{n_{0 b}^{j-k}}{n_{0 t}^{0}}, \\
b_{b}^{j}= & -\frac{e}{m\left[i\left(\omega-\gamma v_{z}\right)+\bar{v}_{e a}\left(v_{0}\right)\right]}\left(\frac{\partial F_{0 b}}{\partial v_{z}}\right)_{z=0} \\
& \times \sum_{k=0}^{j} \frac{(-1)^{k} \bar{\nu}_{e a}\left(v_{0}\right)^{k}}{k ! v_{0}^{k}} E^{0 j-k} .
\end{aligned}
$$

Таким образом, при разложении с точностью до $z^{N}$ мы получаем систему $l=4 \cdot(N+1)$ уравнений, из которых $l_{f}=3 \cdot(N+1)-$ кинетические уравнения Больцмана для коэффициентов разложения амплитуд возмущения ФРЭ и $l_{E}=(N+1)-$ уравнения Пуассона для коэффициентов разложения амплитуды возмущения электрического поля. С помощью $l_{f}$ кинетических уравнений вычисляются правые части $l_{E}$ уравнений Пуассона, и мы получаем линейную однородную систему $l_{E}$ уравнений для $l_{E}$ коэффициентов разложения амплитуды возмущения электрического поля. Система имеет ненулевое решение тогда и только тогда, когда определитель ее матрицы равен нулю. Это условие и есть дисперсионное уравнение для нахождения инкремента затухания (усиления) возмущений в плазме.

Для простоты здесь рассмотрим однородную плазму и однородную изотропно распределенную по направлениям движения группу электронов с энергией пучка, испытавших упругие столкновения. Тогда при $N=0$, т.е. когда предполагается, что невозмущенный пучок имеет постоянную плотность, но в кинетических уравнениях для возмущений ФРЭ всех трех групп учитываются упругие электрон-атомные столкновения, из уравнения
Пуассона (четвертое уравнение системы (П5)), используя первые три соотношения (П5), получаем дисперсионное уравнение в виде

$$
\begin{aligned}
-i \gamma & =I_{b}+\frac{I_{t}}{1-\frac{1}{2} \int_{-1}^{1} a_{t}(v, \mu) d \mu} \\
& +\frac{I_{i b}}{1-\frac{1}{2} \int_{-1}^{1} a_{b}^{i}-(v, \mu) d \mu},
\end{aligned}
$$

где

$$
\begin{gathered}
I_{b}=8 \pi^{2} e \int_{0}^{\infty} \int_{-1}^{1} v^{2} b_{b}^{0}(v, \mu) d \mu d v \\
I_{i b}=8 \pi^{2} e \int_{0}^{\infty} \int_{-1}^{1} v^{2} b_{b}^{i 0}(v, \mu) d \mu d v \\
=-i \gamma \frac{\omega_{i D}^{2} E^{00}}{\gamma^{2} v_{0}^{2}}\left[\frac{(1+\varepsilon)^{2}}{\varepsilon(2+\varepsilon)}-1\right], \\
I_{t}=8 \pi^{2} e \int_{0}^{\infty} \int_{-1}^{1} v^{2} b_{t}^{0}(v, \mu) d \mu d v ; \quad \omega_{i D}^{2}=\frac{4 \pi^{2} e n_{0 b}^{i}}{m} .
\end{gathered}
$$

Уравнение (П6) представляет собой дисперсионное уравнение рассматриваемой системы трех групп электронов в задаче с начальными условиями, когда при известном действительном волновом числе $\gamma$ ищется комплексная частота $\omega$. Для получения дисперсионного уравнения в задаче с граничными условиями необходимо выразить волновое число $\gamma$ через переменные $\omega, \varepsilon$.

\section{Конфликт интересов}

Авторы заявляют, что у них нет конфликта интересов.

\section{Список литературы}

[1] Wang Huihui, V. Sukhomlinov, I. Kaganovich, A. Mustafaev. Plasma Sourc. Sci. Technol., 26, 024002 (7p.) (2017). https://doi.org/10.1088/1361-6595/26/2/024002

[2] Wang Huihui, V. Sukhomlinov, I. Kaganovich, A. Mustafaev. Plasma Sourc. Sci. Technol., 26, 024001 (10p.) (2017). https://doi.org/10.1088/1361-6595/26/2/024001.

[3] В.С. Сухомлинов, А.С. Мустафаев, А.А. Страхова, О. Мурильо. ЖТФ, 87 (12), 1824 (2017). [V.S. Soukhomlinov, A.S. Mustafaev, A.A. Strahova, O. Murilio. Tech. Phys. 62 (12), 1822 (2017). https://doi.org/10.1134/s1063784217120258]

[4] V.S. Sukhomlinov, A.S. Mustafaev, N.A. Timofeev. J. Appl. Phys., 123, 143301 (2018). https://doi.org/10.1063/1.5019819

[5] А. Ахиезер, Я. Файнберг. ДАН, 64, 555 (1949).

[6] D. Bohm, E.P. Gross. Phys. Rev. 75, 1851 (1949). https://doi.org/10.1103/PhysRev.75.1851

[7] Ф.Г. Бакшт, В.Ф. Лапшин. ЖТФ, 61 (6), 13 (1991).

[8] F.G. Baksht, V.F. Lapshin, A.S. Mustafaev. J. Phys. D Appl. Phys., 28, 694 (1995).

https://doi.org/10.1088/0022-3727/28/4/012

[9] F.G. Baksht, V.F. Lapshin, A.S. Mustafaev. J. Phys. D: Appl. Phys., 28, 689 (1995).

https://doi.org/10.1088/0022-3727/28/4/011 
[10] A.S. Mustafaev, A.P. Mezentsev. J. Phys. D: Appl. Phys., 9 (1), L69 (1986). https://doi.org/10.1088/0022-3727/19/5/001

[11] А.С. Мустафаев. ЖТФ, 77 (4), 111 (2001). [A.S. Mustafaev, Tech. Phys., 46 (4), 472 (2001).]

[12] V. Sukhomlinov, R. Matveev, A. Mustafaev, N. Timofeev. Phys. Plasmas, 27 (6), 062106 (2020). https://doi.org/10.1063/5.0001822

[13] V.S. Sukhomlinov, R.J. Matveev, A.S. Mustafaev, N.A. Timofeev, D.Q. Solihov. Phys. Plasmas, 27 (8), 083504 (2020). https://doi.org/10.1063/5.0011584

[14] H.E. Singhaus. Phys. Fluids, 7, 1534 (1964). https://doi.org/10.1063/1.1711408

[15] D. Sydorenko, I.D. Kaganovich, P.L.G. Ventzek, L. Chen. Phys. Plasmas, 23, 122119 (2016). https://doi.org/10.1063/1.4972543

[16] Y. Raizer. Gas Discharge Physics (Springer, Berlin, NY., 1991), p. 536. https://doi.org/10.1007/978-3-642-61247-3

[17] S.A. Self, M.M. Shoucri, F.W. Crawford. J. Appl. Phys., 42 (2), 704 (1971). https://doi.org/10.1063/1.1660084

[18] W.E. Drummond. Phys. Fluids, 7 (6), 816 (1964). https://doi.org/10.1063/1.1711291

[19] S. Pramanik, V.I. Kuznetsov, N. Chakrabarti. Phys. Plasmas, 22, 082103 (2015). https://doi.org/10.1063/1.4927794

[20] Л.Д. Ландау. ЖЭТФ. 16, 574 (1946).

[21] А.И. Ахиезер. Электродинамика плазмы 2-е изд., перераб. (Наука, М., 1974), 720 с.

[22] D. Sydorenko, I.D. Kaganovich, L. Chen, P.L.G. Ventzek. Phys. Plasmas, 22, 123510 (2015). https://doi.org/10.1063/1.4937785

[23] I.D. Kaganovich, D. Sydorenko. Phys. Plasmas, 23, 112116 (2016). https://doi.org/10.1063/1.4967858

[24] D. Sydorenko, I.D. Kaganovich, P.L.G. Ventzek, L. Chen. Phys. Plasmas, 25, 011606 (2018). https://doi.org/10.1063/1.5018427

[25] V.M. Malkin, N.J. Fisch. Phys. Rev. Lett. 89 (12), 125004-1 (2002). https://doi.org/10.1103/physrevlett.89.125004

[26] P.H. Yoon, Tongnyeol Rhee, Chang-Mo Ryu. Phys. Rev. Lett. 95, 215003 (2005). https://doi.org/10.1103/Phys. Rev. Lett.95.215003

[27] W.E. Drummond. Phys. Fluids, 7 (6), 816 (1964). https://doi.org/10.1063/1.1711291

[28] W.E. Drummond, D. Pines. Annals Phys., 28, 478 (1964). https://doi.org/10.1016/0003-4916(64)90205-2

[29] L.M. Al'tshul', V.I. Karpman. Sov. Phys. JETP, 22 (2), 361 (1966).

[30] В.П. Тараканов, Е.Г. Шустин. Физика плазмы. 7 (2), 151 (2007).

[31] А.А. Серов. Физика плазмы. 35 (7), 624 (2009).

[32] N.G. Matsiborko, N. Onishchenko, D. Shapiro, V.I. Shevchenko. Plasma Phys., 14 (2), 5911972. https://doi.org/10.1088/0032-1028/14/6/003

[33] S.A. Self, M.M. Shoucri, F.W. Crawford. J. Appl. Phys., 42 (2), 704 (1971). https://doi.org/10.1063/1.1660084

[33] M. Adibzadeh, C.E. Theodosiou. Atomic Data and Nuclear Data Tables. 91, 8 (2005).

https://doi.org/10.1016/j.adt.2005.07.004 